\title{
EL DESARROLLO DE LA SINTAXIS POSTERIOR
}

\author{
JESÚS ROSEL \\ (Universidad de Málaga)
}

\section{II: EN LA DESCRIPCION DE UN PERSONAJE ${ }^{1}$}

\section{Introducción}

Cada vez está tomando más importancia en psicología el estudio de los procesos del lenguaje humano; e incluso, entre los lingüistas, del mismo modo, se tiene más conciencia de que las teorías gramaticales remiten a un sistema de comportamiento humano. Como ya indicara Chomsky (1975, p. 61); "La teoria del lenguaje es simplemente esa parte de la psicología humana que trata de un "órgano mental» particular: el lenguaje humano".

Es preciso reconocer que no todas las teorías lingüisticas son tan «mentalistas» como la generativista de Chomsky, ni todas las teorias psicológicas atribuyen igual importancia al estudio del lenguaje; pero desde los años 60 (en gran parte debido a la influencia de Chomsky) se han multiplicado los estudios interdisciplinarios sobre psicología y lenguaje.

En este artículo encuadraremos los resultados del desarrollo de la «sintaxis posterior" dentro de varios aspectos cognitivos planteados como antinomias (o hipótesis explicativas de carácter contrapuesto) existentes en psicología del lenguaje: autonomia versus funcionalidad del lenguaje; capacidad de competencia versus actuación lingüística; predominio de la cognición versus predominio del lenguaje en el desarrollo humano.

1 El presente trabajo forma parte de un estudio más general, cuya primera parte ha sido publicada bajo el título «El desarrolio de la sintaxis posterior. I: En el relato de narración de historias», en Anales de Filología Hispánica, 3, 1987, pp. 83-105. 


\subsection{Sintaxis autónoma versus sintaxis funcional}

La hipótesis de que la producción de habla (y fundamentalmente de la sintaxis) es de carácter autónomo ha sido formulada en la teoría del lenguaje actual por Chomsky; y de hecho, la mayor parte de las investigaciones llevadas a cabo sobre este aspecto han seguido su influencia. Chomsky mantiene la posición de que existe un dispositivo de producción del lenguaje como un sistema independiente del resto del comportamiento humano (Chomsky, 1974, 1975). Todavía en sus últimas publicaciones mantiene que el hombre está formado por distintas facultades cognitivas con principios de actuación diferentes y con estructuras propias (Chomsky, 1979, 1980, 1982).

Los psicolingüistas defensores de la autonomia de la sintaxis siguen en sus planteamientos la hipótesis de Chomsky con más o menos fidelidad: Garret, 1976, 1980; Fay, 1980 a, 1980 b; Flores d'Arcis, 1982. Casi todas las investigaciones de estos autores están hechas con pruebas de laboratorio y sobre sujetos adultos, pero sin poner apenas en correspondencia los aspectos sintácticos del contenido del experimento con otros más comprehensivos de los mismos sujetos que hacian las pruebas en el laboratorio (como su capacidad cognitiva, la función comunicativa de la tarea propuesta, etc.).

La hipótesis funcionalista plantea que el lenguaje es sólo uno de los aspectos del comportamiento humano, y que tanto en la comprensión como en la expresión del habla no se recurre únicamente a estrategias lingüísticas, sino que también se utilizan procesos cognitivos, comunicativos y contextuales para clarificar el mensaje.

Dentro de la corriente «funcionalista» (aunque en otros aspectos teóricos difieran entre sí notablemente) se agrupan psicolingüistas con clara influencia conductista (Le Ny, 1979; Osgood, 1980), piagetana (Sinclair, 1978; Oleron, 1979; KarmiloffSmit, 1979), cognitivista (Clark, 1978; Slobin, 1979) o de procesamiento de la información (Anderson, 1980; Ellis, 1980; Bock, 1982).

En sus escritos, los partidarios de la hipótesis autonomista en el procesamiento de la sintaxis explicitan de manera inequivoca (generalmente) su posición respecto al debate. No obstante, los teóricos que mantienen la alternativa funcionalista de la sintaxis no suelen plasmar abiertamente su hipótesis (tal vez porque éstos son mayoría, no forman una escuela definida, y porque la hipótesis funcionalista goza de una tradición más obvia dentro de las corrientes psicolingüísticas).

Cada enfoque (autonomista o funcionalista) sobre la producción de la sintaxis tiene sus peculiaridades y sus ventajas metodológicas:

a) Desde la posición funcionalista, la explicación de la producción sintáctica (o lingüística) puede ser relacionada con los aspectos evolutivos del ser humano, pero también dicha evolución general se enriquecería si los funcionalistas detallasen con más precisión las propiedades del lenguaje que investigan.

b) Los autonomistas suelen ser más rigurosos en la formalización de las muestras de habla que estudian, especificando cuáles son las propiedades intrínsecas del lenguaje y sus supuestos mecanismos de producción.

Resumiendo, puede decirse que los funcionalistas estudian el habla insistiendo en los aspectos psicológicos y comunicativos del lenguaje, mientras los «autono- 
mistas" lo hacen procurando destacar los rasgos puramente lingüisticos y formales de las emisiones.

Quizás el planteamiento antitético entre la psicolingüistica autónoma y la funcional es excesivamente esquemático, pero es un buen exponente de la situación, aunque hubiese que identificar en cada autor (sobre todo en los funcionalistas) los aspectos que contiene su propia teoría acerca de los dos enfoques señalados. Cada uno de estos enfoques implica una serie de hipótesis respecto a nuestra investigación que se plantearán en el epígrafe 2 de este artículo.

\subsection{Competencia versus actuación individual del lenguaje}

En 1965 (trad. cast.: 1975), Chomsky revisó su teoría de 1957 (trad. cast.: 1974) sobre la producción de la sintaxis, planteando nuevas hipótesis de carácter cognitivo respecto al habla humana. Uno de los aspectos que formula Chomsky en 1965 es el de la dicotomía entre la capacidad de competencia y de actuación del hablante.

Aunque la dicotomía "competencia-actuación" (en sus aspectos sintácticos) ha influido (directa o indirectamente) en varios psicolingüistas, también ha provocado gran cantidad de críticas, que agruparemos bajo tres epígrafes:

1. La "competencia» es una abstracción epistemológica, separada de la realidad, y que, por tanto, supone una idealización filosófica que no puede ser refutada ni corroborada en el plano de los hechos (Hörmann, 1982).

2. Hay autores que anteponen (metodológicamente) el estudio de la actuación al de la competencia para elaborar una teoría gramatical, defendiendo que (en todo caso) ambos aspectos interactúan, pues la capacidad de competencia es formulada por el lingüista a partir de un conjunto de actuaciones. En esta línea, han criticado a Chomsky Oller (1970) y, en general, casi todos los teóricos de la lingüistica pragmática y de la del texto (Schmidt, 1977; Van Dijk, 1983).

3. Un tercer grupo de críticos insiste en que la competencia (como máximo nivel teórico de realización de la actuación humana) no tiene por qué ser sintáctica. Así, Lakoff (1970) señala que la competencia no sería sintáctica, sino de tipo semántico; Campbell y Walles (1975) indican que la competencia humana puede ser de diferentes tipos, y Piaget (1973) mantiene que la competencia lingüística radica en la capacidad lógico-operatoria de los sujetos.

De cualquier forma, la teoría de la dicotomía competencia-actuación en el comportamiento humano ha sido asumida implícitamente por distintos psicólogos cognitivistas, aplicándola al estudio de la memoria humana (Anderson, 1976), la capacidad de procesamiento (Pylynshyn, 1978) y el desarrollo infantil (Piaget, 1975).

\subsection{Predominio de la cognición versus predominio del lenguaje en el desarrollo humano}

Sin pretender ser exhaustivos, señalaremos que el conductismo (en su planteamiento más radical) asume que el pensamiento es un subproducto del lenguaje, o 
que en todo caso el pensamiento sería un habla subvocal (Watson, 1972; Skinner, 1981). Dicha teoría sobre las relaciones entre lenguaje y cognición está prácticamente desechada.

Aunque la mayoría de los autores consideran que el lenguaje y la cognición interactúan de manera funcional, es preciso reseñar también la teoría de Piaget de que el lenguaje, en su ontogénesis, está supeditado a la cognición. Básicamente, son dos los argumentos que Piaget aduce sobre la dependencia del lenguaje respecto a la cognición:

a) A lo largo del desarrollo, el niño precisa (como requisito indispensable) de una determinada capacidad lógico-cognitiva para adquirir determinados aspectos del lenguaje. Así, el niño ha de poseer la noción de permanencia del objeto antes de utilizar las primeras palabras (Piaget, 1975); igualmente, otros investigadores de la escuela de Ginebra han comprobado que un niño ha de estar en el nivel de la operatividad concreta para usar correctamente términos relacionales y descripciones coordinadas (Sinclair, 1978); también el niño ha de poseer ese nivel para expresar adecuadamente secuencias de carácter temporal (Ferreiro, 1971).

b) Piaget mantiene la teoría de que la lógica operatoria (de acción reflexiva sobre los objetos) es el origen del desarrollo cognitivo (independientemente del lenguaje) y a dicho desarrollo lógico quedarian supeditadas las demás estructuras de comportamiento.

Además, según Piaget, para conseguir el máximo nivel lógico-cognitivo (el de la operatividad formal), sería necesario el uso del lenguaje porque permite desligar las operaciones de los objetos con que se efectúan; pero esto no sería suficiente pues las operaciones formales (una vez adquiridas) desbordan el lenguaje y exigen un lengtłaje propio: el de la lógica matemática (Piaget, 1973).

\section{Hipótesis y procedimiento de investigación}

\subsection{Hipótesis}

Las hipótesis planteadas guardan relación con cada antinomia teórica expuesta anterriormente y serán formuladas mediante un procedimiento "crucial»:

- Por lo que concierne a la antinomia autonomía versus funcionalidad del desarrollo sintáctico, si se cumple la hipótesis autonomista, las oraciones más complejas (en dificultad de utilización y en profundidad) no tienen por qué aparecer correlacionadas con el desarrollo cognitivo (apreciado a través de la prueba de lógica relacional) ni con la edad; pero, por el contrario, si es cierta la hipótesis funcional, habrá una correlación entre el desarrollo del lenguaje con la cognición y con la edad del niño.

- Respecto a las antinomias sobre la competencia versus la actuación y la referida al predominio de la cognición versus el lenguaje, hemos de señalar que si se cumple la hipótesis de la competencia cognitiva, para que el niño llegue a utilizar las expresiones sintácticas más complejas, es preciso que antes posea una determi- 
nada capacidad cognitiva. En el caso contrario, ha de admitirse como más coherente la hipótesis de la actuación (pragmática) ${ }^{2}$.

\subsection{Metodología}

La metodología utilizada es la misma que en el epígrafe 2 de nuestro trabajo sobre la sintaxis en la narración de historias ${ }^{3}$. En el presente artículo se utilizan como variables dependientes:

- La complejidad sintáctica en la descripción de un personaje (V4), calculada siguiendo el mismo procedimiento que en la narración de historias (V2), pero V4 se aplica al "corpus" de descripción de un personaje.

- La profundidad sintáctica en la descripción de un personaje (V5), utilizando el mismo sistema que $V 3$ aplicándose al relato de descripción de un personaje.

(En el Anexo de este artículo se adjunta un ejemplo de operacionalización de un relato de descripción de un personaje.)

\section{Resultados}

Los resultados brutos conseguidos en el relato de descripción de un personaje se exponen en el cuadro número 1 (para los niños) y en el número 2 (para las niñas).

Las hipótesis planteadas anteriormente sobre el desarrollo de la sintaxis en el relato de descripción han sido comprobadas mediante el cálculo de correlación ordinal de Spearman. Los resultados numéricos aparecen en el cuadro número 3. Dichos cálculos corresponden al total de la muestra (niños y niñas).

En el cuadro número 3 se puede comprobar cómo la complejidad sintáctica en la descripción correlaciona positivamente con la edad del relator. No ocurre así con la correlación entre la complejidad sintáctica en la descripción de un personaje y las respuestas de lógica dadas por el niño.

No obstante, la profundidad en la descripción de un personaje correlaciona positivamente con la edad y con el número de respuestas correctas en la prueba de lógica.

Por consiguiente, la variable dependiente más significativa a efectos de desarrollo infantil es la referida a la profundidad sintáctica. También en la prueba de narración de historias la variable dependiente "profundidad sintáctica» (V3) es la que correlaciona con las variables independientes (edad y capacidad lógica) ${ }^{4}$.

Por lo que respecta a los resultados cuantitativos (y cualitativos simultáneamente), se comprueba que hay un orden de adquisición de las subordinaciones sintácticas (que dependen más de la edad que del nivel lógico) en el contexto de relato de descripción de un personaje. Puesto que la prueba (tal como está plan-

2 Aunque ha sido expuesta, no se plantea la hipótesis del predominio del lenguaje sobre la cognición.

3 Vid. Jesús Rosel, «El desarrollo de la sintaxis posterior. I: En el relato de narración de historias», cit.

4 Cfr. Ibid. 
CUADRO N. ${ }^{\circ} 1$

Resultados obtenidos por los niños en la prueba de «descripción de un personaje»

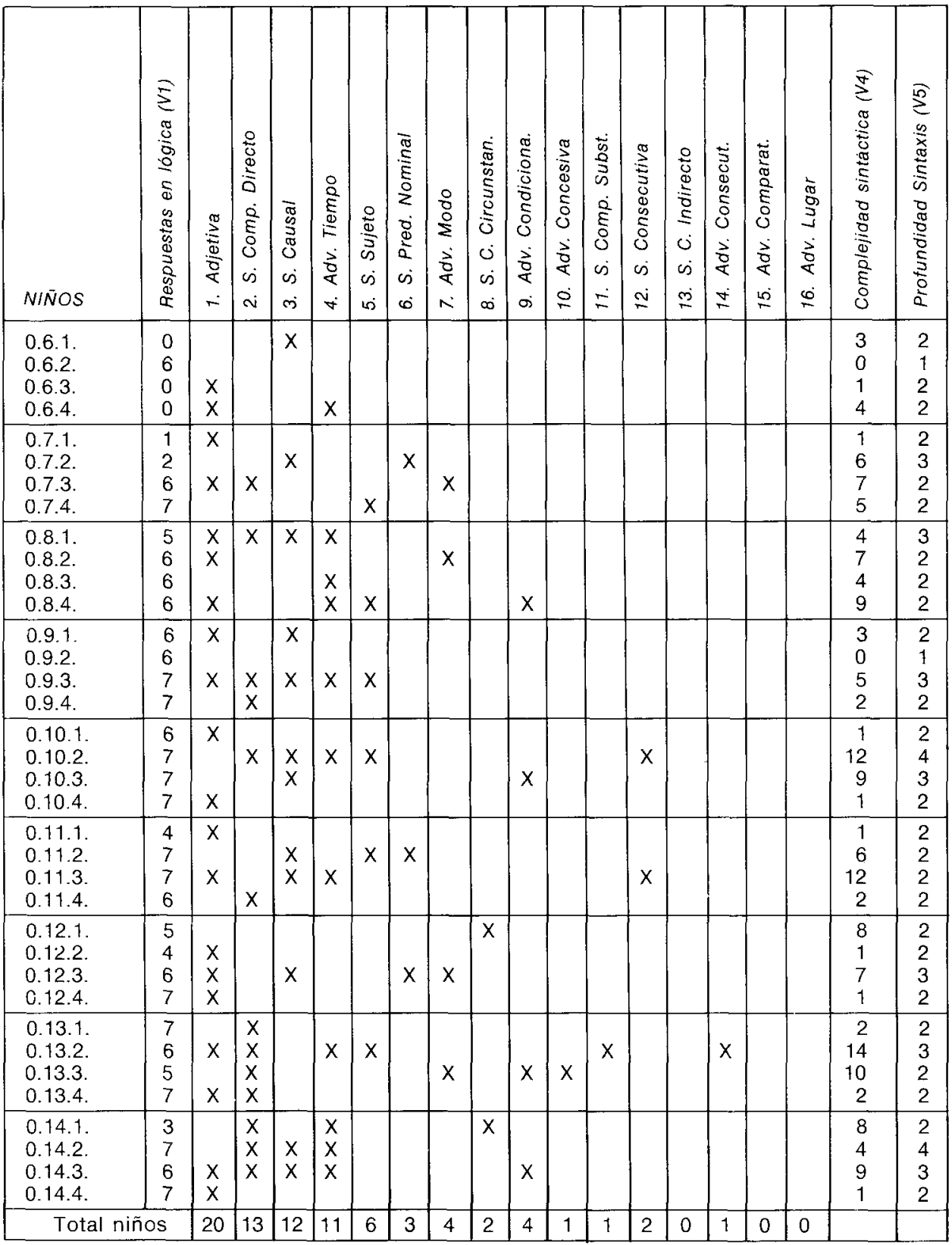


CUADRO N. ${ }^{\circ} 2$

Resultados obtenidos por los niñas en la prueba de «descripción de un personaje»

\begin{tabular}{|c|c|c|c|c|c|c|c|c|c|c|c|c|c|c|c|c|c|c|c|}
\hline NIÑAS & $\begin{array}{l}\vec{F} \\
\mathbb{8} \\
0 \\
0 \\
0 \\
0 \\
0 \\
0 \\
\mathbb{8} \\
0 \\
0 \\
0 \\
0 \\
0 \\
0 \\
0\end{array}$ & $\frac{\substack{0 \\
\frac{0}{0}}}{4}$ & 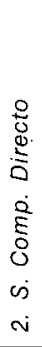 & 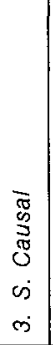 & 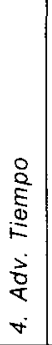 & $\begin{array}{l}\frac{0}{0} \\
\frac{9}{5} \\
\omega \\
\omega \\
\text { is }\end{array}$ & 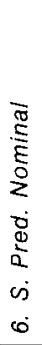 & $\begin{array}{l}\circ \\
8 \\
\circ \\
\Sigma \\
\dot{8} \\
\dot{8} \\
ن\end{array}$ & 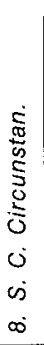 & 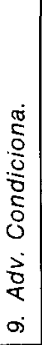 & 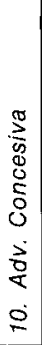 & 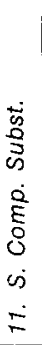 & 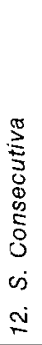 & 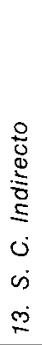 & 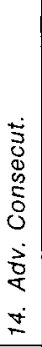 & 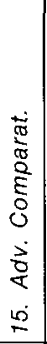 & 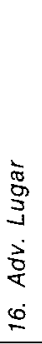 & 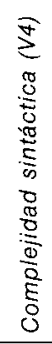 & $\begin{array}{l}10 \\
2 \\
0 \\
0 \\
0 \\
5 \\
5 \\
0 \\
0 \\
0 \\
0 \\
5 \\
3 \\
0 \\
0\end{array}$ \\
\hline $\begin{array}{l}\text { A. } 6.1 . \\
\text { A. } 6.2 \text {. } \\
\text { A. } 6.3 \text {. } \\
\text { A.6.4. }\end{array}$ & $\begin{array}{l}0 \\
0 \\
1 \\
6\end{array}$ & & $x$ & & & $\begin{array}{l}x \\
X\end{array}$ & & $x$ & & & & & & & & & & $\begin{array}{l}7 \\
5 \\
5 \\
2 \\
\end{array}$ & $\begin{array}{l}2 \\
2 \\
2 \\
2\end{array}$ \\
\hline $\begin{array}{l}\text { A.7.1. } \\
\text { A. } 7.2 \text {. } \\
\text { A. } 7.3 \text {. } \\
\text { A. } 7.4 \text {. }\end{array}$ & $\begin{array}{l}0 \\
0 \\
3 \\
3\end{array}$ & $\begin{array}{l}x \\
x\end{array}$ & $x$ & $x$ & & & $x$ & & & & & & & & & & & $\begin{array}{l}0 \\
0 \\
6 \\
1\end{array}$ & $\begin{array}{l}1 \\
1 \\
4 \\
2\end{array}$ \\
\hline $\begin{array}{l}\text { A.8.1. } \\
\text { A.8.2. } \\
\text { A.8.3. } \\
\text { A.8.4. }\end{array}$ & $\begin{array}{l}6 \\
5 \\
5 \\
3 \\
\end{array}$ & & $x$ & $x$ & $x$ & $x$ & & & & & & & & $x$ & & & & $\begin{array}{r}13 \\
3 \\
0 \\
0\end{array}$ & $\begin{array}{l}3 \\
3 \\
1 \\
1\end{array}$ \\
\hline $\begin{array}{l}\text { A.9.1. } \\
\text { A.9.2. } \\
\text { A.9.3. } \\
\text { A.9.4. }\end{array}$ & $\begin{array}{l}6 \\
6 \\
6 \\
4\end{array}$ & $\begin{array}{l}x \\
x\end{array}$ & $x$ & & & & $x$ & & $x$ & $x$ & & & & & & & & $\begin{array}{l}9 \\
0 \\
0 \\
6\end{array}$ & $\begin{array}{l}2 \\
1 \\
1 \\
2\end{array}$ \\
\hline $\begin{array}{l}\text { A. } 10.1 \\
\text { A. } 10.2 \\
\text { A. } 10.3 \\
\text { A. } 10.4\end{array}$ & $\begin{array}{l}7 \\
6 \\
6 \\
7\end{array}$ & $x$ & $\begin{array}{l}X \\
X \\
X \\
x\end{array}$ & $\begin{array}{l}x \\
x\end{array}$ & $\begin{array}{l}x \\
x\end{array}$ & & $x$ & $x$ & $\begin{array}{l}x \\
x\end{array}$ & & & $\begin{array}{l}X \\
X\end{array}$ & & & & & & $\begin{array}{r}4 \\
11 \\
11 \\
8\end{array}$ & $\begin{array}{l}3 \\
3 \\
3 \\
4\end{array}$ \\
\hline $\begin{array}{l}\text { A.11.1. } \\
\text { A.11.2. } \\
\text { A.11.3. } \\
\text { A.11.4. }\end{array}$ & $\begin{array}{l}3 \\
6 \\
6 \\
7\end{array}$ & $x$ & $\begin{array}{l}x \\
x\end{array}$ & $x$ & & $x$ & & & & $x$ & $\begin{array}{l}x \\
x\end{array}$ & & & & & & & $\begin{array}{r}2 \\
10 \\
0 \\
10\end{array}$ & $\begin{array}{l}2 \\
2 \\
1 \\
3\end{array}$ \\
\hline $\begin{array}{l}\text { A.12.1. } \\
\text { A.12.2. } \\
\text { A.12.3 } \\
\text { A. } 12.4\end{array}$ & $\begin{array}{l}7 \\
7 \\
5 \\
3\end{array}$ & $\begin{array}{l}x \\
x\end{array}$ & $x$ & & $x$ & $x$ & & & & & & & & & & & & $\begin{array}{l}0 \\
2 \\
5 \\
1 \\
\end{array}$ & $\begin{array}{l}1 \\
2 \\
2 \\
2\end{array}$ \\
\hline $\begin{array}{l}\text { A.13.1. } \\
\text { A.13.2 } \\
\text { A.13.3. } \\
\text { A.13.4. }\end{array}$ & $\begin{array}{l}6 \\
6 \\
6 \\
7\end{array}$ & $\begin{array}{l}x \\
x \\
x\end{array}$ & $\begin{array}{l}x \\
x \\
x\end{array}$ & $\begin{array}{l}x \\
x \\
x \\
x\end{array}$ & $x$ & $x$ & & & & & $x$ & $x$ & & & & & & $\begin{array}{r}10 \\
3 \\
11 \\
5\end{array}$ & $\begin{array}{l}4 \\
2 \\
4 \\
3\end{array}$ \\
\hline $\begin{array}{l}\text { A. } 14.1 . \\
\text { A.14.2. } \\
\text { A. } 14.3 \text {. } \\
\text { A. } 14.4\end{array}$ & $\begin{array}{l}7 \\
6 \\
7 \\
7\end{array}$ & $\begin{array}{l}x \\
x\end{array}$ & $\begin{array}{l}x \\
x \\
x\end{array}$ & $\begin{array}{l}x \\
x\end{array}$ & $x$ & $x$ & $x$ & $x$ & $x$ & & $x$ & $x$ & & & & & & $\begin{array}{r}8 \\
10 \\
11 \\
2\end{array}$ & $\begin{array}{l}4 \\
2 \\
3 \\
2\end{array}$ \\
\hline \multicolumn{2}{|c|}{ Total niñas } & 13 & 17 & 11 & 6 & 7 & 4 & 3 & 4 & 2 & 4 & 4 & 0 & 1 & 0 & 0 & 0 & & \\
\hline \multicolumn{2}{|c|}{ T. niños+niñas } & 43 & 30 & 23 & 17 & 13 & 7 & 7 & 6 & 6 & 5 & 5 & 2 & 1 & 1 & 0 & 0 & & \\
\hline
\end{tabular}


CUADRO N. ${ }^{\circ} 3$

Coeficientes de correlación ordinal de Spearman entre las distintas variables de la descripción de un personaje

\begin{tabular}{|l|c|c|}
\hline & $\begin{array}{c}\text { Complejidad } \\
\text { sintáctica (V4) }\end{array}$ & $\begin{array}{c}\text { Profundidad } \\
\text { sintáctica (V5) }\end{array}$ \\
\hline Edad & $0,252(\mathrm{p}<5 \%)$ & $0,348(\mathrm{p}<1 \%)$ \\
\hline Respuestas en lógica & 0,218 (N.S.) & $0,376(\mathrm{p}<1 \%)$ \\
\hline $\begin{array}{l}\text { Tras cada índice de correlación se indica entre paréntesis si ese índice no es } \\
\text { significativo (N.S.), o si es significativo a más del } 5 \% \text { o del } 1 \% \text { de probabilidad. } \\
\left.\text { (Para } n=72: r_{c}(5 \%)=0,233 ; r_{c}(1 \%)=0,302\right) .\end{array}$ \\
\hline
\end{tabular}

teada) es de «actuación» lingüística ${ }^{5}$ y que aparece en cada niño una tendencia a repetir cada subordinación utilizada, se trazará el orden de aparición de las subordinaciones sintácticas en función de la edad y del nivel lógico de los dos niños con menor valor en cada una de estas variables.

El orden de aparición de la sintaxis en la descripción de un personaje sería el siguiente:

1. La subordinación adjetiva es emitida (una o varias veces) por 43 niños (59'7\% de la muestra). Los dos primeros niños en utilizarla tienen un nivel lógico de 0 , y son de 6 años de edad.

$2 .^{\circ}$ La oración sustantiva de complemento directo es utilizada en la prueba de descripción de un personaje por 30 niños $(416 \%)$. Aparece primeramente en dos niños de nivel lógico 3 (las niñas A.6.4 y A.11.1), y los dos niños de menor edad que la utilizan tienen 6 años (A.6.4) y 7 años de edad (O.7.3).

$3 .^{\circ}$ La oración subordinada sustantiva causal aparece en 23 niños (31'9\% del total), siendo, por tanto, la tercera en frecuencia en la descripción de un personaje. Es utilizada por dos niños con nivel lógico 0 y 2, de 6 y 7 años de edad (son los niños 0.6 .1 y 0.7 .2 .

$4 .^{\circ}$ La cuarta subordinación, en frecuencia de uso, en la descripción de un personaje es la adverbial de tiempo, siendo utilizada por 17 niños (23'6\%). Los dos niños con menor nivel lógico que la usan consiguen un nivel de 0 respuestas (el niño 0.6.4) y 3 respuestas (el niño 0.14.1); los dos niños con menor edad que ya la utilizan tienen 6 y 8 años de edad.

5. La oración subordinada sustantiva de sujeto aparece a continuación, utilizándose por 13 niños (18\%). La utilizan dos niñas de 6 años de edad, de nivel lógico 0 y 1.

5 En pruebas de relatos, es prácticamente imposible garantizar que cada niño utiliza el máximo de sus posibilidades lingüísticas, por lo que se trata de pruebas de «actuación" antes que de "competencia". Aunque con una muestra de población considerablemente amplia, sería lógico suponer que los máximos valores de «actuación" conseguidos se aproximarían bastante a la «competencia» lingüistica de tipo pragmático. 
6. La subordinación sustantiva de predicado nominal es emitida por 7 niños $(97 \%)$. Los dos más jóvenes en emplearla tienen 7 años de edad, y los dos niños de menor nivel lógico tienen 2 y 3 en la prueba lógica.

7. La subordinación adverbial de modo se utiliza por 7 niños $(9,7 \%)$. La usan ya una niña de 6 años y un niño de 7 años. Los dos mínimos niveles lógicos en los que aparece son de 0 y de 5 .

8. La subordinación que aparece después es la sustantiva de complemento circunstancial, que la emiten 6 niños $\left(8^{\prime} 3 \%\right)$. Los dos más jóvenes tienen 9 y 10 años, y los dos mínimos niveles lógicos de los niños que la utilizan son de 3 y de 5 .

9. La oración adverbial condicional también es usada por 6 niños (8'3\%). Los dos más jóvenes tienen 8 y 9 años, y los dos niños con menor nivel lógico tienen 5 y 6 respuestas correctas.

$10 .^{\circ}$ Posteriormente, aparece la oración adverbial concesiva, utilizada por 5 niños $\left(6^{\prime} 9 \%\right)$. De ellos, los dos más jóvenes son de 11 años, y los dos de mínimo nivel lógico tienen 5 y 6 respuestas en lógica.

11. La subordinada sustantiva complemento de sustantivo es emitida por 5 niños $\left(6^{\prime} 9 \%\right)$. Los dos niños más jóvenes en su utilización tienen 10 años. Mientras los dos con más reducido nivel lógico tienen 6 respuestas de lógica.

12. La oración sustantiva consecutiva aparece en los relatos de descripción de 2 niños (2'7\%), de 10 y 11 años de edad, de nivel lógico 7 .

$13 .^{\circ}$ La oración sustantiva de complemento indirecto es utilizada por un solo niño $(1 ' 3 \%)$ de 8 años de edad y nivel lógico de 6 .

$14 .^{\circ}$ La subordinación adverbial consecutiva es emitida por un niño $(1 \% 3 \%)$ de 8 años de edad y nivel lógico de 6.

Las oraciones subordinadas adverbiales comparativas y adverbiales de lugar no son úilizadas por ningún niño en la prueba de descripción de un personaje.

Respecto a la profundidad sintáctica en la descripción, el valor máximo conseguido es de 4 . Los niños más jóvenes que han conseguido una profundidad sintáctica de 4 , tienen 7 y 10 años de edad (0.10.2 y A.7.3); de entre los niños que consiguen la profundidad de 4 , los dos con menor nivel lógico tienen 3 y 6 respuestas correctas en lógica.

Si se compara la máxima profundidad de la descripción de un personaje (cuatro niveles) con la máxima de la narración, se comprobará que en éste último contexto la puntuación más alta es de 6. Da la impresión de que la narración admite mayor número de encadenamientos en la subordinación, pero este aspecto será contrastado con más detalle en la tercera sección de este estudio.

\section{Conclusiones}

Como se comprueba, la capacidad sintáctica (en complejidad y en profundidad de subordinaciones) en una prueba de descripción de un personaje se desarrolla en el niño entre los 6 y 14 años en función de dos variables: primera, la edad del niño y, segunda, el nivel lógico del niño.

En nuestro trabajo ya citado sobre la narración comprobábamos una mayor correlación entre el nivel lógico y la capacidad sintáctica que entre la edad y la capacidad sintáctica. De cualquier modo, nuestros resultados confirman la hipótesis 
de que la capacidad sintáctica sigue una evolución por encima de los 6 años, habiendo subordinaciones que no aparecen hasta una edad relativamente tardía y cuando el niño tiene una capacidad lógica bien desarrollada.

En lo concerniente a la hipótesis sobre autonomia-funcionalidad de la sintaxis, los resultados tienden a confirmar la mayor verosimilitud de la hipótesis funcional: la capacidad sintáctica (en complejidad y en profundidad) por correlacionar con la edad y el nivel lógico del niño supone que los aspectos referidos a la psicolingüística de la sintaxis evolucionan conjuntamente con otros aspectos del desarrollo (la edad y la cognición).

El mismo Chomsky ha flexibilizado últimamente algo su posición inicial respecto a la autonomía de la sintaxis. Así en 1980 considera que la mente está estructurada modularmente, o sea, como un «sistema de subsistemas interaccionados que poseen sus específicas propiedades especiales» (Chomsky, 1980, p. 89), lo cual puede interpretarse como una aproximación a las teorías funcionalistas del lenguaje humano.

De cualquier forma, tal como Chomsky planteó originariamente su modelo de la autonomía sintáctica, aunque ha sido adoptado como hipótesis de trabajo de una corriente importante de psicolingüistas, es preciso puntualizar que se trata (principalmente) de una hipótesis filosófico-lingüistica antes que estrictamente psicológica. Da la impresión de que Chomsky se ha preocupado más por especificar cuáles son las bases formales del funcionamiento del lenguaje que de relacionar dichas bases formales con otras variables del desarrollo y del comportamiento humano. Ahora bien, los presupuestos de los que parte la lingüística no tienen por qué ser idénticos a los de la psicología.

Respecto a la antinomia competencia-actuación, hemos de señalar que en la descripción de un personaje las subordinaciones sintácticas más complejas —la 11, sustantiva complemento de sustantivo; la 12, sustantiva consecutiva; la 13 , sustantiva de complemento indirecto; y la 14, adverbial consecutiva- son utilizadas sólo por niños con una capacidad lógica de 6 o 7 respuestas lógicas. Algo semejante ocurre con la profundidad sintáctica. Así, dado un nivel de desarrollo lógico de un niño relator, podria señalarse cuál sería el máximo nivel sintáctico (en profundidad y en complejidad) que alcanzaria el niño ${ }^{6}$. Resumiendo, puede establecerse una competencia cognitiva y sintáctica, de la cual se infiere hasta qué nivel puede llegar un niño, pero esa competencia se establece a partir de la máxima actuación.

La tercera antinomia está establecida en torno a la polémica respecto al dominio de la cognición versus del lenguaje en el desarrollo humano. Los resultados obtenidos en nuestra investigación corroboran la hipótesis de Piaget acerca del predominio de la cognición sobre el lenguaje.

Ahora bien, en cuanto al desarrollo ontogenético tampoco puede negarse lo contrario. Posiblemente, el lenguaje constituye una forma especial de actuación (vicariante) del sujeto sobre el medio, acción que estaría acompañada de una reflexión sobre la realidad. Es decir, en terminología piagetana, la actividad lingüística del niño puede considerarse como una «operación interiorizada», que, a su vez,

6 Recuérdese que una prueba de relatos no es en sí misma una prueba sobre la “competencia» lingüística. 
favorecería el desarrollo lógico-cognitivo del hablante. Por consiguiente, el lenguaje (en su evolución) no estaría totalmente supeditado a la lógica, ni tampoco la desbordaría, sino que ambas facultades interaccionarian y se coordinarían en la actuación del sujeto, siendo ambas la manifestación de una capacidad cognitiva más global para el individuo.

\section{III: EN FUNCION DE LOS CONTEXTOS DE ENUNCIACIÓN}

\section{Introducción}

Una vez que se ha comprobado la importancia de las variables de sujeto en la evolución de la sintaxis, se comprobará cómo infuyen las variables de tarea, por medio de los contextos de narración en los que han de expresarse los sujetos.

Inicialmente, puede señalarse que la narración de una historia y la descripción de un personaje son dos tipos (relativamente diferentes) de relatos orales que pueden ser solicitados a un niño. Un psicólogo diría que son dos tipos de tareas distintas, mientras un lingüista afirmaría que se trata de dos textos diferentes.

Ahora bien, ha de reseñarse que el texto producido por un emisor depende del contexto de enunciación (en un sentido amplio). Como indica Lyons (1983, p. 197) «texto y contexto son complementarios: cada uno de ellos presupone al otro". No obstante, cuando el mismo Lyons intenta hacer una definición de lo que es el contexto llega a la conclusión de que «no se puede dar una respuesta simple a la pregunta ¿qué es el contexto?" (Lyons, 1983, p. 220).

Pese a la pregunta (sin respuesta unívoca) de Lyons, intentaremos realizar una aproximación teórica al contexto como una variable (de tarea o de situación) que afecta a la realización del habla. Para ello, se hará una somera revisión de lo que es el contexto según sea estudiado por la lingüística, la antropología, la sociología o la psicología. Así se considerará el contexto desde tres perspectivas: a) desde el punto de vista de la lingüística; b) como situación de relación interpersonal «cara a cara» donde las condiciones del receptor (según la edad, el status, etc.) pueden afectar a las características de la emisión del hablante, y c) como condiciones del ambiente (inmediato o amplio) que cooperan a orientar el lenguaje de un hablante hacia unos contenidos que, a su vez, influyen en las estructuras formales del lenguaje.

1.a) Slama-Cazacu (1970) entiende como contexto al «resto de palabras que acompañan a una palabra», restringiendo el contexto gramatical a las relaciones de tipo paradigmático entre las palabras de un decurso de habla.

Históricamente, la lingüistica estructural ha sido la teoría que más importancia ha concedido al contexto (explícita o implícitamente) como conjunto de relaciones entre palabras. Así, Saussure (en 1916) manifiesta que cualquier unidad lingüística establece dos tipos de relación, en función de las probabilidades de aparición en un decurso: las sintagmáticas y las asociativas (Saussure, 1973). Las relaciones sintagmáticas en terminología de Hjelmslev (1969), que es más usada, se producen entre unidades que pueden aparecer en esa misma unidad.

Esta idea del contexto como relación ha influido en la psicología del lenguaje y 
cognitiva entre varios investigadores que han estudiado la formación de conceptos: Werner y Kaplan (1950), Rosch (1978) y Bruner (1980), entre otros.

En los últimos años, la noción de contexto ha sido ampliada por los lingüistas del texto (Van Dijk, 1983; Kintsch, 1979; y Schmidt, 1977, entre otros), así como por los lingüistas interesados por la pragmática (Searle, 1980), de modo que el contexto se convierte en «una abstracción de lo que intuitivamente llamaríamos situación comunicativa» (Van Dijk, 1983, p. 81). Así, los elementos del contexto serían los "que determinan sistemáticamente la aceptación (o no), el logro (o fracaso) o la idoneidad (o no) de los enunciados".

Se comprueba, entonces, cómo la noción de contexto ha sido ampliada por los gramáticos hasta incluir elementos que conciernen también al estudio de la psicología y de la sociología.

1.b) El lenguaje ha sido estudiado empíricamente en situaciones «cara a cara» entre dos o más personas (sobre todo cuando una de ellas es un niño), según la relación de familiaridad o conocimiento-desconocimiento de los interlocutores. Así, el contexto viene definido por las variables interpersonales de la situación. Este sistema de estudio del contexto según la interacción ha sido estudiado por psicólogos infantiles, psicólogos sociales y sociólogos.

En este epígrafe destacan los estudios sobre la interacción lingüística madreniño (Brow y Bellugi, 1972; Fraser y Roberts; 1975; Martlew, 1980). También se ha investigado la capacidad lingüística del niño según sea el receptor a quien se dirige: la madre, un niño de edad aproximadamente igual a la del emisor, un niño más pequeño, un muñeco, un adulto extraño, etc. (Sachs y Devin, 1976; Snow, 1978). Igualmente, los sociólogos han estudiado la comunicación establecida según el status de dos hablantes adultos pertenecientes a distinto nivel social (Labov, 1964).

Todos estos investigadores demuestran que existen diferencias en los estilos de lenguaje usados por un mismo individuo (sea éste niño o adulto), procurando éste adaptarse a las características cognitivas del interlocutor, al contexto físico, a la tarea requerida, al medio social, etc.

1.c) En el epígrafe anterior se entendía el contexto como una característica del interlncutor (principalmente). En este tercer epígrafe se estudiará el lenguaje como instrumento de comunicación que se aplica a diferentes contenidos conceptuales.

Malinowsky, en 1923, resaltó la importancia del lenguaje como manifestación que se produce en un «contexto de situación» y que a la vez es una actividad especial realizada junto a otras actividades (Malinowsky, 1964). Este autor (aunque fue antropólogo) ha ejercido una notable influencia sobre la que se ha dado en llamar la escuela lingüística de Londres, entre cuyos miembros destacan Firth, HaIliday, Lyons y Robins.

En una línea de investigación más semejante a la nuestra, Weinrich (1974), a partir del análisis de textos escritos, llegó a la conclusión de que existen dos grandes bloques de lenguaje: el del «mundo comentado»y el del «mundo narrado». El primero es fundamentalmente de carácter comunicativo y descriptivo: pedir y dar información, dialogar, pronunciar una conferencia, etc. La función del lenguaje del mundo narrado es informar sobre algo que ya ha transcurrido; en este segundo bloque encuadra el autor los cuentos, las historias, las novelas, etc. Según Weinrich, cada uno de esos grupos de lenguaje exige unas formas y tiempos verbales específicos. 
Edwards (1976) reseña que se utilizan expresiones más largas y más complejas en sus aspectos morfosintácticos cuando la tarea lingüística a realizar es más formal.

Bronckart $(1979,1982)$ ha elaborado una clasificación sistemática de los textos, intentando simultáneamente detectar (mediante su correspondiente análisis), cuáles son los rasgos morfológicos y morfosintácticos (él los denomina marcas) propios de cada tipo de texto.

Las investigaciones y las teorias revisadas aquí vienen a corroborar que no existe un habla individual única (en el sentido de actuación personal o de estilo propio de habla). De este modo se ha relativizado el valor de la gramática normativa tradicional, potenciándose el estudio de la pragmática lingüística.

El objetivo de la pragmática, tal como Morris lo planteaba ya en 1938, sería el estudio de la relación entre los signos lingüísticos y sus usuarios o intérpretes (Morris, 1962). Dicha definición tan amplia, ha sido modificada y detallada por otros autores. Así Stalnaker (en Schmidt, 1977, p. 41) indica que «la pragmática es el estudio de los actos lingüísticos y de los contextos en los que aquéllos se usan". Dicha definición se ciñe con más precisión a lo que actualmente se entiende como pragmática, conteniendo la pragmática dos dominios: a) el de la efectividad en la comunicación (por ejemplo: el de la teoría de los «actos del habla»), y b) el de la producción de textos en función del contexto de enunciación.

En este artículo los contextos de enunciación oral son considerados como dos variables de tarea diferente: 1. la narración de una historia, y 2. la descripción de un personaje. El tipo de diseño es «intrasujeto", pues cada niño realiza las dos tareas. El objetivo ha sido la comprobación de si cada uno de estos dos contextos afecta a la sintaxis utilizada por el niño en el relato.

\section{Hipótesis y metodología}

Las hipótesis sobre los contextos son las siguientes:

- Se comprobará si existen diferencias significativas, a nivel intrasujeto, en el uso de la sintaxis (tanto en la variable dependiente complejidad sintáctica como en la profundidad sintáctica) entre el contexto de narración de una historia y el contexto de descripción de un personaje.

- Se comprobará si existe correlación significativa entre la puntuación obtenida por un mismo niño, entre la complejidad sintáctica de la narración y de la descripción; igualmente, se comprobará si hay correlación entre la profundidad sintáctica de la narración y de la descripción.

- Se comprobará si el orden de aparición de los diferentes tipos de subordinaciones sintácticas, en la narración y en la descripción, es el mismo. Para ello se calculará la correlación ordinal (intercontextos) de la frecuencia de uso de las subordinaciones sintácticas entre la narración y la descripción.

La muestra de población, las pruebas pasadas a los sujetos y el sistema de operacionalización de las variables han sido los mismos que ya se han utilizado en las dos partes anteriores sobre la sintaxis.

La única salvedad que ha de tenerse en cuenta es que en este trabajo sobre los contextos se han reajustado los datos referidos a la complejidad sintáctica (recuér- 
dese que la puntuación en la variable complejidad sintáctica se operacionalizaba según la frecuencia de aparición de cada tipo de subordinación en cada uno de los dos relatos). Así, puesto que la complejidad sintáctica se cuantificaba en función de la frecuencia de aparición en cada uno de los dos contextos, en este artículo se operacionaliza la complejidad sintáctica según la frecuencia de utilización de la sintaxis en los dos contextos (en total). Por ello, en el cuadro número 4 se reseña la puntuación de la complejidad, sumando la frecuencia de cada contexto y asignando rangos a la suma de dichas frecuencias.

\section{CUADRO N. ${ }^{\circ} 4$}

Rangos asignados a cada tipo de subordinación en función del contexto

\begin{tabular}{|c|c|c|c|c|}
\hline (1) & (2) & (3) & Subordinación & Rango \\
\hline 56 & 43 & 99 & Adjetiva & $1 . \circ$ \\
\hline 46 & 30 & 76 & Sustantiva complemento directo & $2 .^{\circ}$ \\
\hline 29 & 23 & 52 & Sustantiva causal & $3 .^{\circ}$ \\
\hline 25 & 17 & 42 & Adverbial de tiempo & $4 .^{\circ}$ \\
\hline 12 & 6 & 18 & Sustantiva compl. circunstancial & $5,5^{\circ}$ \\
\hline 5 & 13 & 18 & Sustantiva de sujeto & $5,5^{\circ}$ \\
\hline 9 & 5 & 14 & Sustantiva compl. de sustantivo & $7 .^{\circ}$ \\
\hline 5 & 7 & 12 & Adverbial de modo & $8 .^{\circ}$ \\
\hline 5 & 6 & 11 & Adverbial condicional & $9 .^{\circ}$ \\
\hline 8 & 1 & 9 & Sustantiva compl. indirecto & $10 .^{\circ}$ \\
\hline 2 & 5 & 7 & Adverbial concesiva & $11,5^{\circ}$ \\
\hline 0 & 7 & 7 & Sustantiva predicado nominal & $11,5^{\circ}$ \\
\hline 3 & 1 & 4 & Adverbial consecutiva & $13 .^{\circ}$ \\
\hline 1 & 2 & 3 & Sustantiva consecutiva & $14 .^{\circ}$ \\
\hline 1 & 0 & 1 & Adverbial comparativa & $15,5^{\circ}$ \\
\hline 1 & 0 & 1 & Adverbial de lugar & $15,5^{\circ}$ \\
\hline
\end{tabular}

(1) Número de niños que utiliza cada tipo de subordinación en la narración de una historia.

(2) Número de niños que utiliza cada tipo de subordinación en la descripción de un personaje.

(3) Suma total de frecuencias $(1)+(2)=(3)$.

\section{Resultados}

Los resultados obtenidos por los niños y las niñas de la muestra, después de adecuar los datos de la variable complejidad sintáctica, están reseñados en el cuadro número 5 .

Debido a que se trata de un diseño de tipo «intrasujeto», pues cada niño contaba una historia y describia un personaje, la contrastación de los resultados obtenidos en cada una de las dos pruebas anteriores se ha calculado mediante la prueba «T» de Wilcoxon-White (ver Domenech, 1977). Los resultados de los cálculos aparecen en el cuadro número 6 . 
CUADRO N. ${ }^{\circ} 5$

Resultados de la variable "complejidad sintáctica" obtenidos por cada niño en el contexto de narración y de descripción, reorganizando los «rangos» de frecuencia de utilización de cada tipo de subordinación (ver cuadro n. ${ }^{\circ}$ )

\begin{tabular}{|c|c|c|c|c|c|}
\hline NIÑOS & $\begin{array}{l}\frac{5}{0} \\
\frac{0}{5} \\
\frac{\pi}{2} \\
\frac{\pi}{2}\end{array}$ & $\begin{array}{l}\frac{5}{0} \\
\frac{0}{0} \\
\frac{2}{5} \\
5 \\
0 \\
0 \\
0\end{array}$ & NIÑAS & $\begin{array}{l}\frac{1}{0} \\
: 0 \\
\frac{0}{0} \\
\frac{\pi}{2} \\
\frac{\pi}{2}\end{array}$ & $\begin{array}{l}5 \\
0 \\
0 \\
0 \\
0 \\
0 \\
0 \\
0 \\
0\end{array}$ \\
\hline $\begin{array}{l}0.6 .1 \\
0.6 .2 \\
0.6 .3 \\
0.6 .4\end{array}$ & $\begin{array}{l}0 \\
0 \\
2 \\
7\end{array}$ & $\begin{array}{l}3 \\
0 \\
1 \\
4\end{array}$ & $\begin{array}{l}\text { A. } 6.1 . \\
\text { A. } 6.2 \text {. } \\
\text { A. } 6.3 \text {. } \\
\text { A. } 6.4 \text {. }\end{array}$ & $\begin{array}{l}2 \\
2 \\
4 \\
4\end{array}$ & $\begin{array}{l}8 \\
5,5 \\
5,5 \\
2\end{array}$ \\
\hline $\begin{array}{l}0.7 .1 \\
0.7 .2 . \\
0.7 .3 . \\
0.7 .4\end{array}$ & $\begin{array}{r}1 \\
3 \\
13 \\
3\end{array}$ & $\begin{array}{c}1 \\
11,5 \\
8 \\
5,5\end{array}$ & $\begin{array}{l}\text { A.7.1. } \\
\text { A. } 7.2 . \\
\text { A.7.3. } \\
\text { A. } 7.4\end{array}$ & $\begin{array}{l}4 \\
2 \\
4 \\
3\end{array}$ & $\begin{array}{c}0 \\
0 \\
11,5 \\
1\end{array}$ \\
\hline $\begin{array}{l}0.8 .1 \\
0.8 .2 \\
0.8 .3 \\
0.8 .4\end{array}$ & $\begin{array}{l}2 \\
2 \\
4 \\
3\end{array}$ & $\begin{array}{l}4 \\
8 \\
4 \\
9\end{array}$ & $\begin{array}{l}\text { A.8.1. } \\
\text { A.8.2. } \\
\text { A.8.3. } \\
\text { A.8.4. }\end{array}$ & $\begin{array}{l}3 \\
2 \\
4 \\
3\end{array}$ & $\begin{array}{r}10 \\
3 \\
0 \\
0\end{array}$ \\
\hline $\begin{array}{l}0.9 .1 \\
0.9 .2 \\
0.9 .3 \\
0.9 .4\end{array}$ & $\begin{array}{l}4 \\
5,5 \\
4 \\
3\end{array}$ & $\begin{array}{l}3 \\
0 \\
5,5 \\
2\end{array}$ & $\begin{array}{l}\text { A. } 9.1 \text {. } \\
\text { A. } 9.2 \text {. } \\
\text { A. } 9.3 \text {. } \\
\text { A. } 9.4 \text {. }\end{array}$ & $\begin{array}{l}5,5 \\
2 \\
2 \\
5,5\end{array}$ & $\begin{array}{c}9 \\
0 \\
0 \\
11,5\end{array}$ \\
\hline $\begin{array}{l}0.10 .1 \\
0.10 .2 \\
0.10 .3 \\
0.10 .4\end{array}$ & $\begin{array}{c}5,5 \\
1 \\
7 \\
11,5\end{array}$ & $\begin{array}{r}1 \\
14 \\
9 \\
1\end{array}$ & $\begin{array}{l}\text { A. } 10.1 \text {. } \\
\text { A. } 10.2 \text {. } \\
\text { A. } 10.3 \text {. } \\
\text { A. } 10.4 \text {. }\end{array}$ & $\begin{array}{r}5,5 \\
5,5 \\
11,5 \\
3\end{array}$ & $\begin{array}{l}4 \\
7 \\
7 \\
5,5\end{array}$ \\
\hline $\begin{array}{l}0.11 .1 . \\
0.11 .2 . \\
0.11 .3 . \\
0.11 .4\end{array}$ & $\begin{array}{l}2 \\
15,5 \\
14 \\
1\end{array}$ & $\begin{array}{c}1 \\
11,5 \\
14 \\
2\end{array}$ & $\begin{array}{l}\text { A. } 11.1 . \\
\text { A. } 11.2 . \\
\text { A.11.3. } \\
\text { A.11.4. }\end{array}$ & $\begin{array}{r}10 \\
9 \\
4 \\
1\end{array}$ & $\begin{array}{c}2 \\
11,5 \\
0 \\
11,5\end{array}$ \\
\hline $\begin{array}{l}0.12 .1 \\
0.12 .2 \\
0.12 .3 \\
0.12 .4\end{array}$ & $\begin{array}{l}1 \\
7 \\
5,5 \\
4\end{array}$ & $\begin{array}{l}5,5 \\
1 \\
8 \\
1\end{array}$ & $\begin{array}{l}\text { A. } 12.1 . \\
\text { A. } 12.2 . \\
\text { A. } 12.3 \\
\text { A. } 12.4 \text {. }\end{array}$ & $\begin{array}{c}4 \\
1 \dot{5}, 5 \\
9 \\
3\end{array}$ & $\begin{array}{l}0 \\
2 \\
5,5 \\
1\end{array}$ \\
\hline $\begin{array}{l}0.13 .1 \\
0.13 .2 \\
0.13 .3 \\
0.13 .3\end{array}$ & $\begin{array}{c}5,5 \\
13 \\
4 \\
4\end{array}$ & $\begin{array}{c}2 \\
13 \\
11,5 \\
2\end{array}$ & $\begin{array}{l}\text { A. } 13.1 . \\
\text { A. } 13.2 . \\
\text { A. } 13.3 . \\
\text { A. } 13.4 \text {. }\end{array}$ & $\begin{array}{r}13 \\
3 \\
4 \\
10\end{array}$ & $\begin{array}{l}11,5 \\
3 \\
7 \\
5,5\end{array}$ \\
\hline $\begin{array}{l}0.14 .1 \\
0.14 .2 \\
0.14 .3 \\
0.14 .4\end{array}$ & $\begin{array}{l}2 \\
1 \\
4 \\
5,5\end{array}$ & $\begin{array}{l}5,5 \\
4 \\
9 \\
1\end{array}$ & $\begin{array}{l}\text { A. } 14.1 \text {. } \\
\text { A. } 14.2 \text {. } \\
\text { A. } 14.3 \text {. } \\
\text { A. } 14.4 \text {. }\end{array}$ & $\begin{array}{l}8 \\
0 \\
8 \\
9\end{array}$ & $\begin{array}{c}5,5 \\
11,5 \\
7 \\
2\end{array}$ \\
\hline
\end{tabular}


CUADRO N. ${ }^{\circ} 6$

Resultados del cálculo « $T$ » de Wilcoxon transformados en valores « $Z$ » con su correspondiente nivel de significación

\begin{tabular}{|c|c|c|}
\hline Valor de «T» & 1.206 & 762 \\
\hline Valor de « $Z »$ & 0,606 (N.S.) & $3,09(\mathrm{p}<0,01)$ \\
\hline & $\begin{array}{c}\text { Contraste en «complejidad } \\
\text { sintáctica» entre narración } \\
\text { y descripción }\end{array}$ & $\begin{array}{c}\text { Contraste en «profundidad } \\
\text { sintáctica» entre narración } \\
\text { y descripción }\end{array}$ \\
\hline
\end{tabular}

CUADRO N. ${ }^{\circ} 7$

Resultados del cálculo de la correlación ordinal «intrasujeto»

\begin{tabular}{|l|c|c|}
\hline & $\begin{array}{c}\text { Complejidad sintáctica en } \\
\text { narración - complejidad } \\
\text { sintáctica } \\
\text { en descripción }\end{array}$ & $\begin{array}{c}\text { Profundidad sintáctica en } \\
\text { narración - profundidad } \\
\text { sintáctica } \\
\text { en descripción }\end{array}$ \\
\hline $\begin{array}{l}\text { Valor de la } \\
\text { correlación }\end{array}$ & 0,207 (N.S.) & 0,174 (N.S.) \\
\hline
\end{tabular}

En el cuadro número 6 se comprueba que hay diferencias significativas en el uso intrasujeto de la "profundidad sintáctica» entre la narración y la descripción. Es decir, un mismo niño tiende a utilizar construcciones sintácticas más profundas en la narración que en la descripción $(p<0$ '01). Se comprueba también que no hay diferencias (a nivel intrasujeto) entre los tipos de subordinación que utiliza un niño en la narración y en la descripción.

Respecto a los resultados sobre la correlación ordinal de Spearman, vienen indicados en el cuadro número 7.

En el cuadro número 7 puede comprobarse que no hay correlación significativa entre la complejidad sintáctica intrasujeto de la narración y de la descripción. Asimismo, tampoco hay correlación entre la puntuación de la profundidad sintáctica intrasujetos de la narración y de la descripción.

El que haya diferencias significativas en el contraste de «T» entre la profundidad sintáctica en la narración y en la descripción, pero que no haya correlación entre esas dos variables, significa que en la narración los niños tienden a utilizar construcciones sintácticas más profundas que en la descripción, si bien un niño que obtenga una elevada puntuación en uno de esos contextos no tiene por qué obtenerlo en el otro (puesto que no hay correlación).

Existe correlación significativa (intercontextos) entre el orden de la frecuencia de utilización de las subordinaciones sintácticas en la narración y el de la descripción ( $r s=0$ 679; $p<0$ 01). Es decir, el orden de frecuencia y de aparición de los diferentes tipos de subordinación es significativamente semejante en ambos contextos. 


\section{Conciusiones}

Las conclusiones más importantes sobre la utilización de la sintaxis infantil en función de los contextos son las siguientes:

- No hay diferencias significativas en el uso de la complejidad sintáctica que hace un mismo niño en la narración y en la descripción, aunque, como se recordará, la complejidad sintáctica correlaciona con la edad (en la descripción) y con la capacidad lógica (en la narración).

- El niño tiende a utilizar construcciones sintácticas más profundas en la narración y en la descripción, con la edad y el nivel lógico del niño.

- No hay correlación intrasujeto en la puntuación obtenida por un niño entre la variable complejidad sintáctica en la narración y en la descripción. Lo mismo ocurre con la profundidad sintáctica.

- Las subordinaciones sintácticas siguen, sensiblemente, el mismo orden de aparición y de uso en la narración y en la descripción. Recuérdese que la correlación ordinal (intercontextos) es significativa.

Por ello, puede inferirse que la variable más sensible al contexto es la «profundidad sintáctica". Debido a que las hipótesis sobre el desarrollo de la sintaxis (según la edad y la capacidad cognitiva del hablante) se cumplen con más frecuencia (aunque no ocurre con todas), resulta que son más significativas las variables de sujeto en el desarrollo sintáctico que las variables de contexto.

Haciendo una rápida revisión de las investigaciones, da la impresión de que el contexto influye más en el vocabulario (Rosel, 1983), en la morfosintaxis (Bronckart, 1982; Clemente, 1984) y en la estructura generativa del texto (Van Dijk, 1983) que en la sintaxis formal, utilizadas en la expresión oral.

Hay aspectos cualitativos a tener en cuenta en ambos contextos (narrativo y descriptivo); así, aparecen cuatro subordinaciones que son emitidas por los niños de nivel lógico igual a cero en ambos contextos:

- La subordinada adjetiva.

- La sustantiva de complemento directo.

- La sustantiva causal.

- La adverbial de tiempo.

Por lo cual resulta coherente suponer que son las subordinaciones más sencillas de comprensión y de emisión por el niño.

En el otro extremo habría que señalar las subordinaciones que son emitidas sólo por niños cuyo nivel lógico es máximo (de 6 ó 7 respuestas correctas en la prueba de lógica):

- La sustantiva consecutiva.

- La adverbial consecutiva.

- La adverbial comparativa.

- La adverbial de lugar.

El resto de subordinaciones tiene una complejidad intermedia y van apareciendo a medida que aumenta la edad y la capacidad lógica del niño (véase el orden de aparición en el cuadro número 4).

Teniendo en cuenta estos resultados cuantitativos y cualitativos, podría hacerse 
una gramática generativa de la sintaxis (en complejidad y profundidad), cuyas entradas del sistema fuesen: $1 .^{\circ}$ ) el nivel cognitivo del hablante, $2 .^{\circ}$ ) la edad de ese mismo niño relator, $\left.3 .^{\circ}\right)$ el contexto sobre el que se habla. Dicha gramática establecería un tope máximo de posibilidades de utilización de unos determinados tipos de subordinación y también de profundidad sintáctica. Dados esos topes máximos, podría establecerse que el niño utilizará dicha complejidad o una menor.

Como aspecto importante, aunque se sale del ámbito de la psicología para entrar en el de la pedagogía, ha de señalarse que los índices obtenidos en este estudio podrían tenerse en cuenta para reeducar alumnos con retraso en el lenguaje (y más concretamente en el de la sintaxis), pues hay un orden de adquisición de las subordinaciones; pero a la vez el reeducador habría de considerar cuál es la capacidad lógica del alumno y, simultáneamente, intentar reeducarla, pues ambas variables se influyen mutuamente.

También podría cuestionarse cuál es la capacidad de asimilación de un texto, por parte de un escolar, en función de la complejidad sintáctica del mismo (en tipos de subordinación y en profundidad), pero para ello sería preciso utilizar un diseño más complejo con control de variables afectando a la comprensión, la memoria y la capacidad de expresión del sujeto.

Por último, nuestra investigación pone de manifiesto que aún queda mucho por descubrir sobre la capacidad expresiva infantil en el “desarrollo segundo» de la sintaxis (de acuerdo con Bronckart y otros, 1983) y que es función de todos los interesados por la evolución psicolingüística del niño en edad escolar el intentar conocer y comprender cómo se desarrolla dicha capacidad tan importante en el comportamiento humano.

\section{Resumen general y consideraciones sobre el desarrollo de la sintaxis posterior}

El objetivo de esta investigación ha sido comprobar cómo evoluciona la capacidad sintáctica del escolar, apreciada a través de dos pruebas de relatos orales: la narración de una historia y la descripción de un personaje.

Con el fin de averiguar si existe relación entre la capacidad sintáctica y la cognitiva, se pasó a cada niño una prueba de lógica relacional.

Al intentar formalizar la capacidad sintáctica de cada niño, surge una dificultad importante: la escasez de investigaciones dedicadas al desarrollo sintáctico en los relatos del niño mayor de seis años. Se ha operacionalizado la sintaxis mediante dos procedimientos: la complejidad sintáctica (según el tipo de subordinaciones sintácticas que utilizaba el niño en su relato) y la profundidad sintáctica (cuantificada por medio del número de oraciones subordinadas encadenadas entre sí).

En resumen, el diseño de investigación tiene tres variables independientes: la edad del niño (de 6 a 14 años), su capacidad lógica (desde 0 a 7 respuestas) y el relato emitido por el niño (de narración de una historia y de descripción de un personaje). Las variables dependientes son: la complejidad y la profundidad sintáctica.

Los hallazgos más importantes han sido:

- La capacidad sintáctica del niño se desarrolla por encima de los seis años de edad. 
- La capacidad sintáctica del niño depende, principalmente, de su capacidad lógica, pero también de su edad y del tipo de relato que emita.

- El orden de aparición y de utilización de los diferentes tipos de subordinaciones sintácticas es sensiblemente semejante en la narración y en la descripción (la correlación, $p<0 ’ 01$ ). Dicho orden, en líneas generales, es el siguiente:

1. ${ }^{\circ}$ Adjetiva

2. ${ }^{\circ}$ Sustantiva de complemento directo

3. ${ }^{\circ}$ Sustantiva causal

4..$^{\circ}$ Adverbial de tiempo

5. Sustantiva de complemento circunstancial

6. Sustantiva de sujeto

7. ${ }^{\circ}$ Sustantiva complemento de sustantivo

8. Adverbial de modo

9. Adverbial condicional

10. Sustantiva de complemento indirecto

11. ${ }^{\circ}$ Adverbial concesiva

12. ${ }^{\circ}$ Sustantiva de predicado nominal

$13 .^{\circ}$ Adverbial consecutiva

14. ${ }^{\circ}$ Sustantiva consecutiva

15. ${ }^{\circ}$ Adverbial comparativa

16. ${ }^{\circ}$ Adverbial de lugar

- Los niños tienden a utilizar construcciones sintácticas más profundas en la narración que en la descripción. Ahora bien, no hay correlación significativa entre la profundidad sintáctica obtenida por cada niño en la narración y en la descripción. Es decir, como grupo, los niños realizan las narraciones sintácticamente más profundas que las descripciones; pero un niño que hace una narración sintácticamente profunda no tiene por qué emitir una descripción sintácticamente profunda (y viceversa).

- Las subordinaciones sintácticas más complejas (en la narración y en la descripción) sólo aparecen cuando el niño tiene una capacidad lógica superior.

Por consiguiente, la sintaxis no es una capacidad autónoma, pues no es independiente de la capacidad cognitiva del hablante ni del contenido sobre el que se habla. Además, la capacidad sintáctica se adquiere gradualmente, consiguiendo sus máximos niveles cuando el niño posee ya una elevada capacidad lógica, a partir de los diez o doce años de edad.

Los datos obtenidos, analizados cualitativamente, apuntan hacia la posibilidad de que la competencia del hablante estaria fundamentada en su capacidad de orden lógico, de modo que ésta marcaría el límite superior de capacidad de emisión sintáctica del niño, pues sólo los niños con elevada capacidad lógica utilizan oraciones subordinadas complejas y encadenan subordinaciones a nivel más profundo.

Hasta un determinado límite, el lenguaje aparece supeditado a la cognición del sujeto (según los resultados obtenidos), pero probablemente exista una interacción entre ambas funciones en la vida cotidiana del niño, de forma que el desarrollo en una de ellas favorezca el desarrollo de la otra.

Respecto de los aspectos metodológicos, han de efectuarse las siguientes precisiones: 
- En esta investigación se ha optado por estudiar únicamente la subordinación sintáctica del lenguaje oral de los relatos infantiles, puesto que es uno de los aspectos menos investigados en psicología del lenguaje y por delimitar el contenido de la recogida de datos. Téngase en cuenta que la investigación es una pregunta concreta a una parcela de la realidad. Convendría profundizar también en otros aspectos afines: en el desarrollo morfosintáctico, en el de la coordinación sintáctica, etc. Seguramente, los hallazgos en estos campos podrían tener diversas aplicaciones: elaboración de textos para escolares (con el fin de que el estilo y la complejidad sintáctica se adecuasen al nivel de desarrollo de los mismos), enseñanza del castellano a extranjeros (aunque también habria de investigarse si se aprende mejor la sintaxis conversacional cuando se enseña siguiendo el orden en el que es emitido por el niño castellanoparlante a lo largo de su desarrollo), etc.

- La complejidad sintáctica se ha operacionalizado en este trabajo mediante criterios cuantitativos empíricos, según la frecuencia de utilización por los niños. Sería preciso investigar qué aspectos influyen en el orden de complejidad sintáctica (además de los cognitivos): semánticos, comunicativos, léxicos, etc.

De cualquier forma, pese a haberse sistematizado la complejidad sintáctica a través de la frecuencia de aparición en los relatos, se comprueba que es un índice adecuado, puesto que es consistente con otras variables de desarrollo: la edad del hablante y su capacidad lógica.

- Hay un problema sobre las características del relato emitido por el niño; queda la duda sobre si su relato es el más completo y complejo que puede elaborar; obviamente, la actuación suele quedar por debajo de la competencia. Este problema es inherente a toda la actuación lingüística del hablante, sobre todo en lo referido a pruebas de textos, pues es razonable suponer que el hablante posee unas capacidades sintácticas y lingüísticas que no explicita totalmente en una prueba de relatos. De cualquier modo, esta dicotomía no se da de manera tan aguda en las pruebas de lógica. Además, en nuestra investigación se comprueba que la capacidad para resolver problemas lógicos es un índice bastante correcto para estimar el nivel de competencia del niño sobre su producción sintáctica. Así, los niños con un nivel cero en respuestas sobre capacidad lógica sólo emiten unos determinados tipos de subordinaciones (la adjetiva, la sustantiva de complemento directo, la sustantiva causal y la adverbial de tiempo), mientras sólo los niños con capacidad lógica elevada (6 ó 7 respuestas correctas) utilizan las oraciones subordinadas más complejas (la adverbial consecutiva, la sustantiva consecutiva, la adverbial comparativa y la adverbial de lugar).

Por último, cabe señalar que aún queda mucho por descubrirse e investigar sobre el desarrollo de la sintaxis del niño mayor de 6 años, siendo este área un buen espacio de colaboración interdisciplinar para la lingüistica y la psicología. 


\section{ANEXO}

En este anexo se exponen literalmente la narración y la descripción de la niña A.13.4. Al. final de cada uno de estos relatos se ha puesto su correspondiente esquema oracional, así como la puntuación obtenida en cada variable.

\section{NARRACIÓN DE HISTORIA}

«Una mañana, unos novios que se $\frac{\text { llamaban José y Lucía, pues tuvieron }}{\mathrm{O}_{2}}$ una discusión por culpa de los celos de José, entonces ella le dijo que era mentira, que todo eso que le habian contado era mentira, y él no se lo quería creer. Entonces le $\frac{\text { puso, }}{\mathrm{O}_{8}}$ le hizo unas pruebas para que viera $\frac{\mathrm{O}_{10}}{\mathrm{O}_{9}}$ que era mentira, y entonces ya se

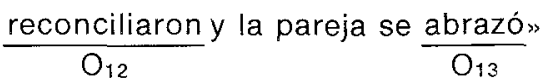

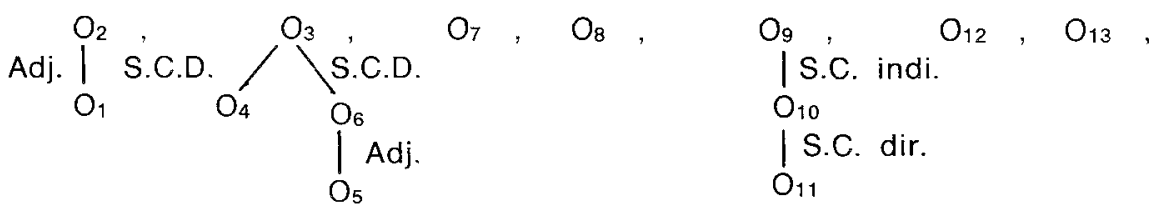

- «Profundidad sintáctica» máxima: 3.

- Subordinaciones que utiliza: 3 (adjetiva, sustantiva de complemento directo y sustantiva de complemento indirecto).

\section{DESCRIPCIÓN DE PERSONAJE}

«Ese José es más bien alto, no es ni gordo ni delgado, corriente, como todas las

$$
\overline{\mathrm{O}_{1}} \overline{\mathrm{O}_{2}}
$$

personas y trabaja en una fábrica. Lo que más le preocupa es su trabajo, llevar bien $\mathrm{O}_{3} \quad \frac{\mathrm{O}_{4}}{\mathrm{O}_{5}}$

a los clientes y hacer bien las cosas, y su novia que no le engañe, y cosas así, porque es muy celoso.

Él está pensando siempre en la fábrica o en su novia, y esto hace que no piense $\frac{\mathrm{O}_{8}}{\mathrm{O}_{9}}$ en las demás cosas». 


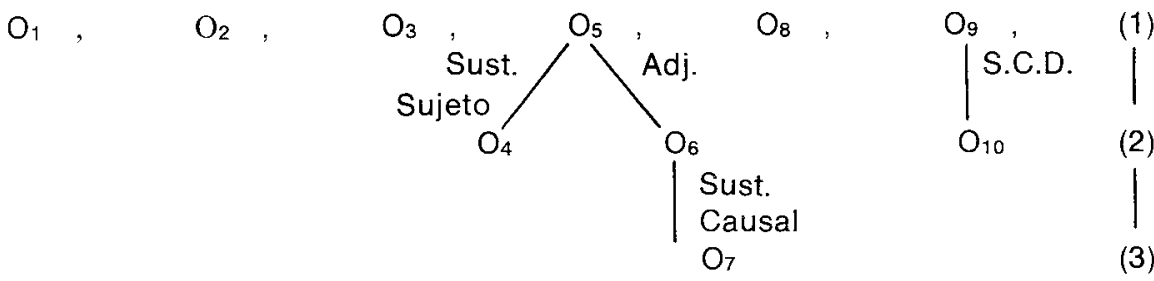

\section{Referencias bibliográficas}

Anderson, J. R. (1976), «Language, memory and thought» Hillsdale, NJ: Laurence Erlbaum Ass.

- (1980), "Cognive psychology and its implications" S. Francisco: Freeman.

Bock, J. K. (1982), "Toward a cognitive psychology of syntax. Information processing contributions to sentence formulation" Psychological Review, 89, 1-47. Bronckart, J. P. (1980), «Pour une methode d'analyse des textes» Bruxelles: P.U.B. Bronckart, J. P. y otros (1982), "Classification de textes pour la rédaction et l'analyse» Genève: Section des Sciences de l'Education.

Bronckart, J. P.; Kail, M. y Noizet, G. (1983), L'avenir de la psycholinguistique de l'enfant. En J. P. Bronckart y otros: «Psycholinguistique de l'enfant» Neuchâtel: Delachaux et Niestlé.

Brown, R. y Bellugi, U. (1972), Three processes in the child's acquisition of syntax En R. Brown (Ed.): «Psycholinguistics» New York: Free Press.

Bruner, J. (Ed.) (1980), «Investigaciones sobre el desarrollo cognitivo» Madrid: Pablo del Río.

Campbell, R. N.; Walles, R. (1975), El estudio de la adquisición del lenguaje, en J.

Lyons: "Nuevos horizontes de la lingüística» Madrid: Alianza.

Clark, E.; Clark, H. H. (1978), Universals, relativity and language processing, en J. H. Greenberg (Ed.): «Universals of human language. Vol. I: Method and theory" Stanford: S.U.P.

Clemente, R. A. (1984), Variaciones en el lenguaje espontáneo infantil. En M. Siguan

(Ed.): «Estudios sobre psicologia del lenguaje infantil» Madrid: Pirámide.

Chomsky, N. (1975), “Aspectos de la teoría de la sintaxis» Madrid: Aguilar.

- (1979), «Reflexiones sobre el lenguaje» Barcelona: Ariel.

- (1980), «Rules and representations» Cambridge, Mass.: Columbia University Press.

- (1982), «Ensayos sobre forma e interpretación» Madrid: Cátedra.

Domenech, J. M. (1977), «Bioestadística» Barcelona: Herder.

Ellis, A. W. (1980a), Speech production and short-term memory, en J. Morton y J. C. 
Marshall (Eds.): "Psycholinguistics. Vol. II: Structures and processes» Cambridge, Mass.: M.I.T. Press.

Fay, D. (1980b), Transformations errors. En V. A. Fromkin (Ed.): «Errors in linguistic performance" New York: Academic.

Ferreiro, E. (1971), «Le relations temporelles dans le langage de l'enfant" Genève: Droz.

Flores d'Arcais, G. B. (1982), "Automatic syntactic computations and use of semantic information during sentence comprehension" Psychological Research, 44, 231-242.

Fraser, C. y Roberts, N. (1975), Mother's speech to children of four different ages. Journal of Psycholinguistic Research, 4, 9-17.

Garret, M. F. (1976), Syntactic processes in sentence production. En R. J. Wales y E. Walker (Eds.): "New approaches to language mechanisms" Amsterdam: North-Holland.

- (1980), Levels of processing in sentence production. En B. Butterworth (Ed.): "Language production. Vol. I: Speech and talk» London: Academic Press.

Hjelmslev, L. (1969), «El lenguaje» Madrid: Gredos.

Hörmann, H. (1982), «Querer decir y entender» Madrid, Gredos.

Karmiloff-Smith, A. (1979), «A functional approach to child language» Cambridge: C.U.P.

Kintsch, W. (1979), "La memoria para prosa». En C. N. Cofer (Ed.): «Estructura de la memoria humana" Barcelona: Omega.

Labov, W.: «Phonological correlates of social stratification» American Antropologist, $66,164-176$.

Lakoff, G. (1970), «Irregularity in syntax» New York: Holt, Rinehart and Winston.

Le Ny, J. F. (1979), "La semantique psychologique" París: P.U.F.

Lyons, J. (1983), "Lenguaje, significado y contexto» Barcelona: Paidós.

Malinowsky, B. (1964), «El problema del significado en las lenguas primitivas» en C. K. Ogden y l. A. Richards: "El significado del significado" Buenos Aires: Paidós (Or.: 1923).

Martlew, M. (1977), "Mother's control strategies in dyadic motherchild conversation» Journal of Psycholinguistic Research, 9, 327-347.

Morris, C. W. (1962), "Signos, lenguaje y conducta» Buenos Aires: Losada (Or.: 1938).

Oleron, P. (1979), «L'enfant et l'acquisition du langage» París: P.U.F.

Oller, J. W. (1970), «Transformational theory and pragmatics. Modern Language Journal, 54, 504-507.

Osgood, C. E. (1980), "Lectures on language performance» New York:SpringerVerlag.

Piaget, J. (1973), El lenguaje y las operaciones intelectuales. En J. Piaget: «Estudios de psicología genética» Buenos Aires: Emecé.

- (1975), «Psicología del niño» Madrid: Morata.

Pylyshynz, W. (1978), "Computational models and empirical constraints» The Behavioral and Brain Sciences, 1, 93-127.

Rosch, E. (1978), Principles of categorization. En E. Rosch y B. Lloyd (Eds.): "Cognition and categorization" New Jersey, Lawrence Erlbaum. 
Rosel, J. (1983), «Niveles cognitivos y desarrollo léxico-sintáctico» Tesis Doctoral. Universidad de Salamanca.

- (1987), «El desarrollo de la sintaxis posterior. I: En el relato de narración de historias", en Anales de Filologia Hispánica, 3, pp. 83-105.

Sachs, J. y Devin, J. (1976), "Young children's use of age appropiate speech styles in social interaction and role-playing" Journal of Child Language, 3, 81-98.

Saussure, F. (1973), "Curso de lingüística general» Buenos Aires: Losada (Or.: 1916).

Schmidt, S. J. (1977), "Teoría del texto" Madrid: Cátedra.

Searle, J. (1980), "Actos de habla» Madrid: Cátedra.

Sinclair, H. (1978), «Adquisición del lenguaje y desarrollo de la mente» Barcelona: Oikos-Tau.

Skinner, B. (1981), “Conducta verbal» México: Trillas.

Slama-Cazaku, T. (1970), "Lenguaje y contexto» Barcelona: Grijalbo.

Slobin, D. I. (1979), "Psycholinguistics» Genview, III.: Scott and Foresman.

Snow, C. (1978), "The conversational context of language learning". En R. N. Campbell y P. Smith (Eds.): "Recent advances in the psychology of language» London: Plenum Press.

Van Dijk, T. A. (1983), «La ciencia del texto» Barcelona: Paidós.

Watson, J. B. (1972), «El conductismo» Buenos Aires: Paidós.

Werner, H. y Kaplan, E. (1950), «Development of word meaning throught verbal context: an experimental study" Journal of Psychology, 29, 251-257. 